if radiation therapy is to be the primary treatment. If the spleen is already enlarged and radiotherapy is to be the primary treatment, then laparotomy may be necessary because of the difficulties in encompassing a large spleen safely within the radiation field. The real question is apparent when one is considering patients who are thought to have stage I and II Hodgkin's disease. If an abdominal operation reveals negative results, in some institutions radiotherapy will be withheld from below the diaphragm. Obviously in this case a serious error would be made if laparoscopy only is used for staging, since it is already known that one-third of perfectly normal-appearing spleens may contain Hodgkin's disease. Therefore, in patients with stage I and II disease whose liver biopsies are negative at laparoscopy, if no further staging is to be done, radiation therapy must be given to the lymph nodes below the diaphragm and to the spleen. Since one would not rely on results of a negative splenic aspiration it need not, and should not, be performed under these circumstances.

At this institute we prefer this approach to the treatment of most patients with stage I and II disease. It should be emphasised that not only is the complication rate for routine splenic aspiration high but a patient with stage I and II disease who has a negative aspirate must still either have his or her spleen irradiated or removed at subsequent laparotomy. My concern is that while routine laparoscopy is to be encouraged as a screening procedure prior to consideration of staging laparotomy, routine aspiration of the spleen is too hazardous to recommend; it should not be included as part of the initial laparoscopy work-up.

V DeVita

Division of Cancer Treatment, National Cancer Institute, Bethesda, Maryland

\section{Medical and surgical emergencies in divers}

SIR,-The problem of serious emergencies occurring in divers under pressure has recently become the subject of concern and was discussed at a conference arranged by the Society of Underwater Technology in London in November 1975 (leading article, 6 December, p 541). In a modern saturation diving system it may be impossible to bring a diver back to atmospheric pressure in less than several days whatever the medical emergency may be Access to the diver may be difficult because of the location of the decompression chamber and because it may take many hours to compress a doctor or rig medical assistant to the ambient pressure. For this reason mobile pressure chambers in which the diver could be transferred from a barge or rig to a shore facility with transfer under pressure arrangements, where the necessary medical attention can be provided, have been proposed. As you pointed out, at the present time there is no combination of helicopter and light-weight chamber available for this kind of transfer, and in any case it seems likely that the need for such an elaborate procedure would only rarely occur.

It seems to us that it would be more profitable to consider what serious medical or surgical emergencies may arise and what measures should be taken to treat such emergencies at the site of operations. For further

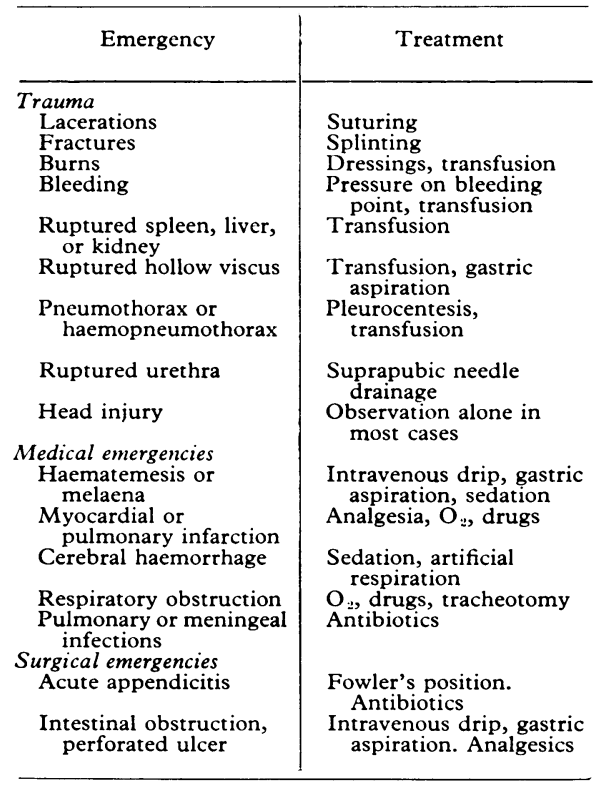

discussion, therefore, we have listed surgical and medical emergencies which we feel are most likely to occur and which would demand urgent treatment, and the treatment of which could be applied in a pressure chamber, if necessary by medical assistants or divers well trained in first aid.

With few exceptions we feel that it should be possible for a well-trained medical assistant or diver to carry out most of these procedures under radio telephone instruction from a doctor, who would carry ultimate responsibility for the treatment. In many cases these procedures would be carried out before the arrival of a medically qualified practitioner, who might then go into the chamber. In our view all but a very small minority of serious emergencies could be effectively dealt with by the application of relatively simple procedures while decompression takes place until transfer to hospital at atmospheric pressure by helicopter is possible.

Department of Industrial Health,

\section{R I McCallum}

University of Newcastle upon Tyn

North Sea Medical Centre,

R A F Cox Gorleston-on-Sea, Great Yarmouth, Norfolk

\section{Radiology and endoscopy in acute upper} gastrointestinal bleeding

SIR,-It has been suggested by Dr K F R Schiller and his colleagues (14 February, $p$ 393) that we have been attacking endoscopy. This has never been our intention, nor could it have been when we have shown that among 61 endoscopy examinations a lesion missed by barium meal was demonstrated in no less than nine cases (31 January, p 270).

Dr Schiller and his colleagues pose four questions to which they are entitled to an answer. Firstly, the relatively small number of cases in our series in a population of some 300000 is because there are four hospitals with acute medical beds serving the area. There was no selection of cases. Secondly, as Dr W Sircus has pointed out (28 February, p 522) all but a few patients were admitted to the general medical wards in this hospital and the decision whether to send the patients for radiology or endoscopy was made by the physician in charge of the case. Thirdly, in the majority of cases radiology was performed first and the barium-meal findings were available to the endoscopist. Fourthly, as $\mathrm{Dr}$ Sircus has very rightly pointed out, the majority of the barium meals were performed within 48 hours of the haemorrhage and the majority of endoscopies after 48 hours, but in those cases where endoscopy and radiology were both performed within the first 48 hours there was no significant difference in the number of positive findings.

Dr Sircus makes a perfectly valid criticism when he states that we, as radiologists, by comparing radiology with endoscopy have set ourselves up as both prosecutor and juryalthough we feel "judge and jury" would be a more appropriate term. Equally, endoscopists who have themselves compared radiology with endoscopy cannot escape similar criticism. With this in mind it is of interest that in a recent study carried out by a radiologist, admittedly in a series containing only a small number of cases of acute upper gastrointestinal bleeding, Cumberland ${ }^{1}$ has shown that a firm diagnosis was made at endoscopy in only 13 of 21 cases $(62 \%)$.

The 10 cases of chronic peptic ulcer in our series which were demonstrated radiologically but not seen at endoscopy must confirm the supplementary nature of the two methods of examination. Surely no one will deny that it is for the physician in charge of the case to decide for himself whether to send for the radiologist or endoscopist and, as Dr W G Scott-Harden has indicated (6 March, p 583), in those hospitals without an endoscopy service it can only be the radiologist.

G M FRASER

D H CUMMACK

$X$-ray Department

Western Genera! Hospital,

Edinburgh

${ }^{1}$ Cumberland, D C, Clinical Radiology, 1975, 26, 223.

\section{Smoking tables for carbon monoxide}

SIR,-The reasons are not clear to me why Dr Wald and his colleagues (21 February, p 434) object so persistently to the view that the carbon monoxide (CO) yields of cigarettes should be included in the Government tar and nicotine tables. When we suggested this in $1973^{1}$ Dr Wald stated that such action then was premature because there was no direct evidence in man implicating $\mathrm{CO}$ as a cause of smoking-related disease. ${ }^{2}$ Now that the case against $\mathrm{CO}$ is stronger Dr Wald and his colleagues object on the grounds that this "would make the tables confusing to smokers who wished to select a less harmful brand, as some cigarettes with high $\mathrm{CO}$ yields have low tar and nicotine yields, and vice versa."

Firstly, this statement is incorrect. None of the brands listed in the Government table's "low tar" group have been shown to have a high CO yield. These brands have ventilated (perforated) filters and their $\mathrm{CO}$ yields are therefore likely to be very low. Furthermore, none of the brands in the "high tar" group have been shown to have $\mathrm{CO}$ yields as low as those of the "low tar" brands.

Secondly, Dr Wald and his colleagues base their conclusion on findings reported in their paper and also presented elsewhere a month before $^{34}$ which suggest that the $\mathrm{CO}$ yields of 
cigarettes with unventilated filters may be a little higher than those of plain cigarettes. But even if some filter-tipped brands do have slightly higher $\mathrm{CO}$ yields than plain cigarettes, it is surely not beyond the intelligence of smokers to choose the ones with the lower CO yields. They could be instructed to pay more attention to tar yields but within each tar yield group to go for the brands with the lower $\mathrm{CO}$ yields. Besides, there are a number of brands which have low tar, low nictotine, and low $\mathrm{CO}$ yields and smokers would have little difficulty in identifying these as the least harmful brands to smoke.

We have found that the $\mathrm{CO}$ yields of different brands of cigarette range from $5 \mathrm{mg}$ to more than $20 \mathrm{mg}$ per cigarette. ${ }^{5}$ Any assessment of the degree of hazard of a particular brand cannot, therefore, be complete without knowledge of its $\mathrm{CO}$ yield. The manufacturers know the $\mathrm{CO}$ yields of their cigarettes, the Government laboratories have already measured them, but the public remain uninformed. It requires only a word from the Secretary of State for Health and Social Security for this important information to be released.

M A H RuSSELL

Addiction Research Unit,

Institute of Psychiatry,

Maudsley Hon

' Russell, M A H, et al, Lancet, 1973, 2, 687.
' Wald, N J, and Smith, P G, Lancet, 1973, 2, 907.

${ }^{2}$ Wald, N J, and Smith, P G, Lance

4 Russell, M A H, Lancet, 1976, 1, 301

${ }^{5}$ Russell, M A H, et al, British Medical fournal, 1975 3,71 .

\section{Laparoscopy explosion hazards with nitrous oxide}

SIR,-Drs G B Drummond and D B Scott (6 March, p 586) have suggested that explosion is not a significant hazard in laparoscopy when nitrous oxide is used unless intestinal gas is released into the peritoneal cavity by puncture of the bowel wall. They carried out analyses of gas samples in 12 laparoscopies only. It is unlikely that the bowel was punctured at all in this small series of cases, but nevertheless there is evidence that there is a significant known incidence of bowel puncture probably of at least $2 \%$. In addition there are a few cases which escape detection at the time of induction of the pneumoperitoneum but which are found by careful routine laparoscopic inspection of the bowel at the conclusion of sterilisation.

The risk of bowel puncture is increased if the Verres needle, which has a locking pin, is employed to enter the abdomen. This needle was originally designed as an all-purpose needle for use in several procedures, including venepuncture, in which the inner cannula needed to be locked outwards. This locking device is not needed by the gynaecological laparoscopist, and if it accidentally becomes locked the risk of bowel puncture is increased. The manufacturers should omit the locking pin, and the inner cannula should also have two side exits for the passage of gas, so reducing the risk of obstruction if the thread of the collar becomes defective.

It is my opinion that nitrous oxide for the pneumoperitoneum should be reserved for those cases in which high-frequency electrical diathermy is not to be used. It has been shown that the minor disturbances caused by carbon dioxide are readily controlled by good general anaesthesia, but that nitrous oxide has some advantages if local anaesthesia alone is employed. For those who prefer to use nitrous oxide always it would be advisable to employ thermal coagulation and division, elastic rings, or clips for sterilisation procedures Even then the occasional bleeding hazard may necessitate rapid use of electrocoagulation.

Patrick Steptoe

Oldham and District General Hospital,

Oldham, Lancs

Use of pressurised aerosols by asthmatic patients

SIR,-It was interesting to read the short reports by Dr J Orehek and others and Drs I C Paterson and G K Crompton (10 January, p 76) concerning the problems associated with the correct use of pressurised aerosols by asthmatic patients. The former study showed that 15 out of 20 patients $\left(75^{\%}\right.$ ) failed either to inspire deeply or hold their breath afterwards, or both, or poorly co-ordinated the puff and the inspiration. In these 15 patients the degree of bronchodilatation achieved after self-administration was significantly smalle than that achieved after administration by the physician. The latter workers identified 45 out of 321 patients $(14 \%)$ as having doubtfully efficient or inefficient techniques as classified by the trained respiratory technician.

In order to obtain objective evidence we monitored the relationship between inspiration and release of the dose in 103 patients attending routine outpatient clinics. A standard pressurised aerosol was modified by the attachment of transducers to provide an electrical analogue of the release of the metered dose and of inspiration. The separate signals were fed to a two-channel recorder. The patient's technique was regarded as satisfactory if the tracing showed that the metered dose was released during inspiration. Thirty-three patients $(32 \%)$ failed to synchronise and the majority of these had been receiving bronchodilator and/or steroid aerosol therapy as part of their treatment.

We are concerned that many patients may not be receiving maximum benefit from these drugs despite initial instruction, and it is now our policy to repeat the tuition at intervals. We have developed a device that detects and indicates, by means of a light, correct synchronisation. The patient practises until he can light the lamp on every attempt.

T J COADY

Chest Department, C J STEWART

pswich Hosp

Ipswich, Suffolk

Medical Department,

H J DAviEs

Allen and Hant
Ware, Herts

\section{Hypertriglyceridaemia and abdominal pain}

SIR,-We read with interest the description by $\mathrm{Dr} \mathrm{R}$ S Elkeles and Mr D Horwell (21 February, $p$ 435) of a case of abdominal pain associated with transient hypertriglyceridaemia and a normal serum amylase level. There have been several recent reports of patients with an elevated serum triglyceride level, abdominal pain, and normal serum amylase activity who were shown at laparotomy to have acute pancreatitis. ${ }^{1}$ Cameron et al $^{2}$ were of the opinion that abdominal pain associated with hypertriglyceridaemia was as indicative of acute pancreatitis as an elevated serum amylase.

Warshaw et al $^{3}$ reported six patients with the clinical features of acute pancreatitis, minimally elevated serum amylase levels, and hyperlipidaemia. The hyperlipidaemic serum was serially diluted and they demonstrated a mean rise in amylase activity of $232 \%$ at 16 dilutions. This is not seen in sera with a high amylase level and normal serum triglyceride levels and suggests that inhibition of the amylase activity in the serum can occur in the presence of an elevated serum triglyceride level.

We have recently seen a patient admitted with abdominal pain whose serum triglyceride level was $112 \mathrm{mmol} / 1$ (9911 $\mathrm{mg} / 100 \mathrm{ml})$ (normal $<2 \mathrm{mmol} / 1(<170 \mathrm{mg} / 100 \mathrm{ml})$ ). The amylase level in undiluted serum (1165 IU/1) was below that acceptable for a diagnosis of acute pancreatitis but rose $338 \%$ to $3936 \mathrm{IU} / \mathrm{l}$, a level diagnostic of acute pancreatitis, when serial dilutions of the serum up to 32 times were performed. Further dilution of the serum resulted in no significant increase in amylase activity, indicating that a plateau had been reached.

It thus appears that in the presence of hyperlipidaemia serial dilutions of serum are necessary to obtain a true serum amylase level. This has obvious importance in the diagnosis of abdominal pain associated with an elevated serum triglyceride level.

L A DONALDSON W McINTOSH

Departments of Surgery and

Biochemistry

Stobhill General Hospital,

Glasgow

Cameron, J L, et al, Surgery, 1971, 70, 53.

Warson, $\mathrm{J}$, et al, Annals of Surgery, 1973, 177, 483 of Surgery, 1975, 182, 72 .

\section{Reticuloendothelial phagocytosis in nephritis}

SIR,-Dr I I Onyewotu (13 March, p 646), together with Dr E J Holborow, has written a classic on the uptake of complexes by macrophages. Nevertheless he is unwise to criticise the in-vivo test without having the appropriate experience. In the long term an in-vivo patient test will be likely to have greater clinical relevance.

The points that he raises may be answered as follows: (1) Only in shock is uptake by the Kupffer cells dependent on hepatic blood flow. In other situations clearance is remarkably independent of liver blood flow. ${ }^{1}$ (2) The free iodide levels in our tests, as we originally stated, ${ }^{2}$ are less than $1 \%$ and can be ignored. Correction is in any case simple. (3) Polyvinylpyrrolidone is certainly not an advantage. Its stated molecular weight is 40000 , which means not only that large amounts will be lost in the urine, but that it is not a macromolecule, only a foreign molecule. The molecular weight of our aggregated albumin is about 760000 . (4) Our patients with mesangiocapillary nephritis show impaired reticuloendothelial system (RES) clearances, as mentioned briefly in our paper (7 February, p 321). This is shown quite clearly by an "RES stress test" that we have used latterly. (5) Dr Onyewotu may be correct in thinking 